\title{
Tipificación de Taraxacum canariense Soest (Asteraceae)
}

\author{
Antonio Galán de Mera
}

\section{Abstract}

GALÁN DE MERA, A. (2012). Typification of Taraxacum canariense Soest (Asteraceae). Candollea 67: 229-232. In Spanish, English and French abstracts.

The name Taraxacum canariense Soest (Asteraceae) is lectotypified. The lectotype corresponds to a plant specimen collected in La Gomera (Canary Islands), deposited at L.

\section{Résumé}

GALÁN DE MERA, A. (2012). Typification de Taraxacum canariense Soest (Asteraceae). Candollea 67: 229-232. En espagnol, résumés anglais et français. Le nom Taraxacum canariense Soest (Asteraceae) est lectotypifié. Le lectotype de Taraxacum canariense, un échantillon récolté à la Gomera (îles Canaries), est conservé à $\mathrm{L}$.

\section{Key-words}

ASTERACEAE - Taraxacum - Typification

Dirección del autor: Departamento de Biología (Botánica), Facultad de Farmacia, Universidad San Pablo-CEU, Apartado 67, E-28660 Boadilla del Monte, Madrid, España. Email: agalmer@ceu.es 


\section{Introducción}

Durante la revisión del género Taraxacum F. H. Wigg. (Asteraceae) para la flora ibérica (CASTROVIEJO, 1986-2012), hemos estudiado todos los taxones citados en Portugal y España, incluyendo las Islas Baleares y Canarias. Muchos de ellos corresponden a endemismos descritos por SoEST (1948, 1951, 1954, 1956, 1961, 1970) sobre material ibérico o canario conservado en ELVE (T. dubium Soest, T. malato-belizii Soest), L (T. balearicum Soest, T. canariense Soest), LISE (T. algarviense Soest, T. duriense Soest, $T$. lusitanicum Soest, T. pinto-silvae Soest, T. triforme Soest) y LOU (T. gallaecicum Soest).

La tipificación correcta de las especies de Taraxacum es muy importante para el conocimiento de las ya descritas, en asociación con el protólogo, con el fin de poderlas comparar con los especímenes de otras nuevas por descubrir. En la mayor parte de las especies de España y Portugal hemos podido encontrar el material tipo, aunque en algunas descritas de Portugal, SoEst lo señaló con una ilustración en el protólogo que coincide con el holótipo elegido, pero sin una designación directa, como ocurre con el material colectado por Werner Rothmaler en Portugal (STAFLEU \& COWAN, 1983) entre 1933 y 1940 (LISE [6417], planta inferior, holótipo de T. duriense; LISE [6437], planta inferior, holótipo de T. algarviense; LISE [6437], planta superior, holótipo de T. triforme; LISE [6465], planta inferior, holótipo de T. lusitanicum). Los casos de $T$. canariense y de $T$. gallaecicum son diferentes, puesto que el protólogo no va acompañado de este tipo de ilustraciones. En T. gallaecicum, Doll (1973) indicó el holótipo sobre un pliego de LOU, pero T. canariense necesita tipificación.

\section{El lectótipo de Taraxacum canariense Soest}

Taraxacum canariense fue publicado a partir de distintos especímenes colectados por Charles-Joseph Marie Pitard en las Islas Canarias.

No conocemos el material de Wageningen al que SOEST alude en el protólogo, pues PitARD \& PROUST (1909) no citaron el herbario WAG en su «Plantae Canarienses Exsiccatae» sino el de Groningen (GRO), cuyas plantas canarias fueron transferidas a L en 1984 (THIERS, 2010). Además, al encontrar material original también en $\mathrm{G}$, elegimos como lectótipo un pliego depositado en el herbario de Leiden.

Taraxacum canariense Soest in Brotéria, Ci. Nat. 23: 139. 1954.

Typus: EsPaña. Islas Canarias: Gomera, Lomo de Fragosa, 1906, C. J. Pitard Pl. Canar. 600 (L!, WAG!).

Lectotypus (designado aquí): EsPaÑa. Islas Canarias: «PLANTAE CANARIENSES, 600 Taraxacum officinale. L./ v. affine Jord./ Gomera: Lomo de Fragoso, in humidis, III.1906», C. J. Pitard Pl. Canar. 600 (L [0533950]; iso-: G [G000 13355]).
Paratypus: EsPaña. Galicia: «Herbarium plantas gallaecicas contineus/ Número.../ Nom. Taraxacum officinale L. var. laevigatum DC./ forma communiforme Rouy/ Habit. ad aggeres viarum prope Arbo... et Humoso», s.d., P. Merino s.n. [LOU].

Soest también utilizó en la descripción de T. canariense Soest material procedente de Galicia, en el NW de España (SoEST, 1954), a juzgar por la información de la etiqueta manuscrita del pliego que se conserva en el herbario LOU; años más tarde, Soest estudió (SAHLIN \& SOEST, 1974) abundante material procedente de Tenerife (L [0533949], [0533951], [0533952], [0533953], [0533954], [0533955], [0533956], [0533957], [0533958], [0533 959], [0533960], [0533961], [0533963]) que, junto con las colecciones originales, nos han servido para aumentar los datos de su descripción:

Planta de hasta $21 \mathrm{~cm}$. Hojas 3-15 x 1-2 cm, espatuladolanceoladas, de pinnatipartidas a pinnatisectas; nervio principal verde, a veces purpúreo; lóbulos laterales 2-5 por hemilimbo, de 2,7-12,6 × 5,5-13 mm, disminuyendo de tamaño hacia el pecíolo - relación entre el largo y el ancho 0,6-1,1 de triangulares a deltoideos, agudos, en ocasiones con el margen distal dentado, recto o convexo; margen proximal de recto a cóncavo, entero, a veces con algún diente interlobular; lóbulo terminal 6,8-29 × 7,4-28,2 mm - relación entre el largo y el ancho 0,6-1 - de triangular a hastado, en ocasiones trilobulado. Escapo de verde a purpúreo, sobre todo hacia la base, erecto y generalmente más largo que las hojas en la antesis, a veces con indumento aracnoideo bajo el capítulo. Capítulo 20,9-34,9 mm de diámetro; involucro 9,8-15,8 × 5,6-9,4 mm; brácteas externas 4,1-7,8 $\times 1,3-2,3 \mathrm{~mm}$, verde-obscuras, purpúreas hacia el ápice, ovado-lanceoladas, de adpresas a reflejas al madurar el capítulo, con borde escarioso hasta de $0,4 \mathrm{~mm}$, frecuentemente cilioladas en los márgenes y el ápice, ecorniculadas; lígulas marginales c. $14 \mathrm{~mm}$, con una banda ancha en el envés de color violeta obscuro; estigmas amarilloverdosos; anteras poliníferas. Aquenios estramíneos; cuerpo 3,1-3,4 mm, con espículas cortas hacia el ápice, el resto rugoso; pirámide $0,5-0,6 \mathrm{~mm}$, de cónica a cilíndrica; rostro 5,9-7 mm; vilano 6,2-7 $\mathrm{mm}$, blanco.

Las brácteas externas del involucro marginadas y ecorniculadas, y la forma de la pirámide nos llevan a incluir a esta especie dentro de la sección Celtica A. J. Richards; no obstante, la parte inferior del cuerpo de los aquenios rugosa la aproximan a T. marklundii Palmgr. (sect. Hamata H. Øllg.). Las dimensiones más pequeñas del involucro de T. canariense, respecto a otras especies de la sect. Celtica, y la estructura de las hojas con los márgenes de los lóbulos frecuentemente convexos, la acercan a $T$. drucei Dahlst., T. leroyi Soest y T. merinoi Soest. Todas ellas forman un grupo de especies que se extienden desde Gran Bretaña, Irlanda, y N y NW de la Península Ibérica hasta la Macaronesia. 


\section{Agradecimientos}

Este trabajo ha sido realizado en el marco del proyecto «Flora iberica VIII» (CGL2008-02982-C03-01/CLI, Ministerio de Ciencia e Innovación, España). Deseamos expresar nuestro agradecimiento a Cornelia Dilger-Endrulat (TUB), Laurent Gautier (G), Francisco Pina (ELVE), Isabel Saraiva (LISE), Javier Silva-Pando (LOU), y a Gerard Thijsse (L), que nos prestaron el material de herbario necesario o nos enviaron información; a los revisores anónimos cuyas opiniones mejoraron el manuscrito inicial.

\section{Referencias}

Castroviejo, S. (1986-2012). Fl. Iber. Madrid.

Doll, R. (1973). Revision der sect. Erythrosperma Dahlst. emend. Lindb. f. der Gattung Taraxacum Zinn. Feddes Repert. 84.

Pitard, J. \& L. Proust (1909). Les Iles Canaries. Flore de l'archipel. Paris.

SAhlin, C. I. \& J. L. van Soest (1974). Two new Taraxaca from the Macaronesian Islands. Agron. Lusit. 35: 313-316.

SoEst, J. L. van (1948). Sur quelques Taraxaca et Hieracia du Portugal. Agron. Lusit. 10: 6-23.

Soest, J. L. van (1951). Sur quelques Taraxaca du Portugal. Agron. Lusit. 13: 67-76.

SoEst, J. L. VAN (1954). Sur quelques Taraxaca de Galice. Brotéria, Ci. Nat. 23: 139-143.

SoEST, J. L. VAN (1956). Nouvelle contribution pour la connaissance des Taraxaca du Portugal. Agron. Lusit. 18: 94-98.

SOEST, J. L. VAN (1961). Quelques nouvelles espèces de Taraxacum, natives d'Europe. Acta Bot. Neerl. 10: 280-306.

Soest, J. L. van (1970). Deux nouvelles espèces de Taraxacum. Melhoramento 22: 83-85.

StAfleu, F. A. \& R. S. CowAN (1983). Taxonomic Literature, vol. 4. Regnum Veg. 110.

ThIERs, B. (2010). Index Herbariorum. New York [http://sweetgum.nybg.org/ih/]. 